\title{
THE VACUUM SYSTEM OF THE LOW ENERGY ION RING AT CERN: REQUIREMENTS, DESIGN, AND CHALLENGES
}

\author{
E. Mahner
}

For the heavy ion program at CERN's Large Hadron Collider, lead-lead collisions with a design luminosity of $10^{27} \mathrm{~cm}^{-2} \mathrm{~s}^{-1}$ are foreseen. This will be achieved after an upgrade of the ion injector chain where the Low Energy Antiproton Ring (LEAR) is currently converted into a Low Energy Ion Ring (LEIR). Avalanche-like heavy-ion induced molecular desorption, first observed at LEAR and systematically studied at CERN's Heavy Ion Accelerator (LINAC 3), is the major challenge to achieve the required average dynamic pressure of about $4 \times 10^{-12} \mathrm{mbar}$ in LEIR. The LEIR vacuum requirements, the technical design, the implementation of non-evaporable getter coatings and low-outgassing collimators, and the concept of ion-beam scrubbing are reviewed in this paper.

CERN, Accelerator Technology Department, Geneva, Switzerland

Paper presented at the 9th European Vacuum Conference

5-7 April 2005, Paris, France

(To be published in Vacuum)

Administrative Secretariat

AT Department

CERN

CH - 1211 Geneva 23
Geneva, Switzerland

25 October 2005 


\title{
The vacuum system of the Low Energy Ion Ring at CERN: Requirements, design, and challenges
}

\author{
E. Mahner \\ CERN, 1211 Geneva 23, Switzerland
}

\begin{abstract}
For the heavy ion program at CERN's Large Hadron Collider, lead-lead collisions with a design luminosity of $10^{27} \mathrm{~cm}^{-2} \mathrm{~s}^{-1}$ are foreseen. This will be achieved after an upgrade of the ion injector chain where the Low Energy Antiproton Ring (LEAR) is currently converted into a Low Energy Ion Ring (LEIR). Avalanche-like heavy-ion induced molecular desorption, first observed at LEAR and systematically studied at CERN's Heavy Ion Accelerator (LINAC 3), is the major challenge to achieve the required average dynamic pressure of about $4 \times 10^{-12} \mathrm{mbar}$ in LEIR. The LEIR vacuum requirements, the technical design, the implementation of non-evaporable getter coatings and low-outgassing collimators, and the concept of ion-beam scrubbing are reviewed in this paper.
\end{abstract}

Keywords: accelerator vacuum system, beam lifetime, cross sections, heavy-ion desorption, nonevaporable getter (NEG), coatings, collimator, scrubbing.

\section{Introduction}

During accelerator operation with heavy ions, large pressure rises have been observed at CERN $[1,2]$, GSI $[3,4]$, and BNL [5,6]. Ions, lost from the beam and striking the vacuum chamber wall, can desorb $10^{4}-10^{5}$ molecules/ion. This effect increases the dynamic pressure and causes further ion losses, which can severely limit the achievable ion intensity and beam lifetime in an accelerator. Dedicated measurements of heavy-ion induced molecular desorption yields have been performed since late 2000 at CERN's LINAC 3, aiming to quantify the desorption effect and to find pragmatic solutions to overcome this potential limitation for the LEIR vacuum system $[2,7,8]$.

\section{LEIR vacuum requirements}

For LEIR, the requested beam lifetime $\tau$ is $30 \mathrm{~s}$. The lifetime due to charge-exchange of a heavy ion with the residual gas is given by

$$
\frac{1}{\tau}=\beta \times c \times \sigma_{t o t} \times n
$$

where $\beta$ is the ion velocity, $c$ the speed of light, $\sigma_{\text {tot }}$ the total cross section, and $n$ the gas density, which does not depend on the gas species. The gas density is given by

$$
n \cong 7.242 \times 10^{24} \times p / T
$$

where the pressure $p$ is measured in mbar and the temperature $T$ in kelvin. At $20^{\circ} \mathrm{C}$ and $1 \mathrm{mbar}$ one finds $n \cong 2.47 \times 10^{22}$ molecules $/ \mathrm{m}^{3}$. In a gas mixture, the product $\sigma_{\text {tot }} \times n$ in equation (1) must 
be replaced by the sum over all relevant gas species, i.e. by $\Sigma \sigma_{\mathrm{i}} \times n_{\mathrm{i}}$. Consequently, the beam lifetime can be expressed in the following way:

$$
\frac{1}{\tau_{\text {total }}}=\frac{1}{\tau_{\mathrm{H}_{2}}}+\frac{1}{\tau_{\mathrm{CH}_{4}}}+\frac{1}{\tau_{\mathrm{CO}}}+\frac{1}{\tau_{\mathrm{CO}_{2}}}+\ldots
$$

To evaluate the LEIR vacuum requirements, we need to calculate the individual cross sections and gas densities for $\mathrm{Pb}^{54+}$ ions with an energy of $4.2 \mathrm{MeV} / \mathrm{u}(\beta=0.0943)$. The charge-exchange of a heavy ion passing through a gaseous medium can be described by the capture and loss of electrons. To determine the electron-capture and electron-loss cross sections ( $\left.\sigma_{\text {capture, }}, \sigma_{\text {loss }}\right)$, formulae introduced by B. Franzke [9] were used. Table I summarizes the calculated cross sections, necessary gas densities, and the equivalent partial pressures for the requested $30 \mathrm{~s}$ beam lifetime in LEIR [10].

\begin{tabular}{lccccc}
\hline \hline Gas & $\sigma_{\text {loss }}\left[\mathrm{cm}^{2}\right]$ & $\sigma_{\text {capture }}\left[\mathrm{cm}^{2}\right]$ & $\sigma_{\text {tot }}\left[\mathrm{cm}^{2}\right]$ & $n\left[\mathrm{~m}^{-3}\right]$ & $\begin{array}{c}p[\mathrm{mbar}] \\
@ 20^{\circ} \mathrm{C}\end{array}$ \\
\hline $\mathrm{H}_{2}$ & $5.38 \times 10^{-19}$ & $1.58 \times 10^{-17}$ & $1.63 \times 10^{-17}$ & $7.22 \times 10^{+11}$ & $2.92 \times 10^{-11}$ \\
$\mathrm{He}$ & $5.38 \times 10^{-19}$ & $1.58 \times 10^{-17}$ & $1.63 \times 10^{-17}$ & $7.23 \times 10^{+11}$ & $2.93 \times 10^{-11}$ \\
$\mathrm{CH}_{4}$ & $2.66 \times 10^{-18}$ & $7.79 \times 10^{-17}$ & $8.06 \times 10^{-17}$ & $1.46 \times 10^{+11}$ & $5.91 \times 10^{-12}$ \\
$\mathrm{H}_{2} \mathrm{O}$ & $2.60 \times 10^{-18}$ & $7.63 \times 10^{-17}$ & $7.89 \times 10^{-17}$ & $1.49 \times 10^{+11}$ & $6.03 \times 10^{-12}$ \\
$\mathrm{~N}_{2}$ & $3.65 \times 10^{-18}$ & $1.07 \times 10^{-16}$ & $1.11 \times 10^{-16}$ & $1.06 \times 10^{+11}$ & $4.29 \times 10^{-12}$ \\
$\mathrm{CO}$ & $3.64 \times 10^{-18}$ & $1.07 \times 10^{-16}$ & $1.11 \times 10^{-16}$ & $1.07 \times 10^{+11}$ & $4.33 \times 10^{-12}$ \\
$\mathrm{Ar}$ & $4.09 \times 10^{-18}$ & $1.20 \times 10^{-16}$ & $1.24 \times 10^{-16}$ & $9.50 \times 10^{+10}$ & $3.84 \times 10^{-12}$ \\
$\mathrm{CO}_{2}$ & $5.71 \times 10^{-18}$ & $1.67 \times 10^{-16}$ & $1.73 \times 10^{-16}$ & $6.81 \times 10^{+10}$ & $2.76 \times 10^{-12}$ \\
\hline
\end{tabular}

Table I: Electron-loss ( $\left.\sigma_{\text {loss }}\right)$ and electron-capture ( $\left.\sigma_{\text {capture }}\right)$ cross sections, the dynamic gas density $(n)$ and corresponding partial pressures $(p)$ for a 30 s beam lifetime of $\mathrm{Pb}^{54+}$ ions at 4.2 MeV/u in LEIR.

One can see that a dynamic pressure (in presence of a circulating lead ion beam) of $4 \times 10^{-12}$ mbar $\mathrm{N}_{2}$ equivalent will be necessary. Assuming $\mathrm{H}_{2}$ is the dominant residual gas, a total pressure of $3 \times 10^{-11}$ mbar would be sufficient for LEIR. A more realistic gas distribution has been measured during dedicated ion-induced desorption experiments at LINAC 3. It was found that the bulk of the molecules desorbed due to lead ion impact was $\mathrm{CO}[7,8] . \mathrm{CO}_{2}$ and $\mathrm{H}_{2}$ were the second most abundant species in the desorbed gas. Taking measured values for the gas composition under heavy ion bombardment, an average dynamic pressure of $\approx 4 \times 10^{-12} \mathrm{mbar}$ is necessary around the LEIR ring for $\tau=30 \mathrm{~s}$ [10]. An error bar for the dynamic pressure cannot be given because this value depends on the achievable gas composition during LEIR operation.

\section{Vacuum system design}

The LEIR vacuum system requires ultrahigh vacuum (UHV) technologies that had been developed at CERN for the Intersecting Storage Rings (ISR) and the Large Electron Positron Collider (LEP). In particular, a very strict choice of materials, vacuum pumps and instrumentation, cleaning procedures, and a sophisticated bakeout system are necessary to reduce the total dynamic outgassing rate to the $10^{-13} \mathrm{mbar} \cdot \ell \cdot \mathrm{s}^{-1} \cdot \mathrm{cm}^{-2}$ range with a negligible amount of leaks. 


\subsection{Vacuum chambers and tanks}

The LEIR vacuum chambers and tanks are made out of stainless steel AISI 316LN or 316L, most of them have been recovered from the former LEAR machine. Due to the UHV requirements, a bakeable all-metal vacuum system with Conflat ${ }^{\mathrm{TM}}$ flanges is used for most machine elements. Wheeler flanges with silver-coated copper wire-seals were chosen for large diameter tanks like kickers and septa. All vacuum chambers and bellows were cleaned according to the CERN procedure [11] and subsequently vacuum fired at either $950^{\circ} \mathrm{C}$ for $2 \mathrm{~h}$ or $600^{\circ} \mathrm{C}$ for $24 \mathrm{~h}$.

\subsection{Pumping system, instrumentation, and bakeout}

Since the dynamic vacuum is a challenge in LEIR, a very careful design of the distribution of pumps and vacuum diagnostics (gauges, residual gas analysers) has been undertaken. A scenario to put linear pumping in terms of non-evaporable getter (NEG) was considered for all parts of the LEIR machine and was implemented wherever possible. Standard and even special shaped vacuum chambers were coated with a $2 \mu \mathrm{m}$ thick TiZrV film and kept under vacuum as long as possible before installation. The layout of the LEIR vacuum system is displayed in Figure 1 .

The vacuum system of the main ring $(\ell \cong 80 \mathrm{~m})$ with its injection $(\ell \cong 12 \mathrm{~m})$ and extraction $(\ell \cong 12 \mathrm{~m})$ lines is separated into five sectors using all-metal type valves. The evacuation from atmospheric pressure to about $10^{-8} \mathrm{mbar}$ is carried out with mobile pumping groups consisting of an all-metal valve, a $200 \mathrm{\ell} / \mathrm{s}$ turbo molecular pump backed with a $30 \mathrm{~m}^{3} / \mathrm{h}$ primary pump, and a control system. The same pumping groups are used for leak detection and during bakeout of a vacuum sector. About $90 \%$ of the injection and extraction vacuum chambers are coated with NEG. For the LEIR main ring vacuum system about $42 \%$ could be NEG coated. Sputter ion

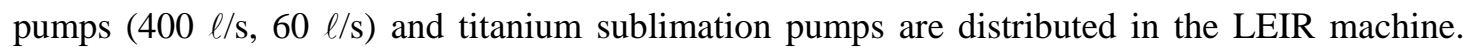
The sublimators are only installed at places where a NEG coating was not feasible, e.g., in the four bending magnet vacuum chambers, the kicker and septa tanks.

For instrumentation, vacuum gauges with a low X-ray limit and low outgassing are the only option for LEIR. We have chosen the CERN-type Bayard-Alpert gauge, developed in the 1970's for the ISR vacuum system [12]. This gauge can reliably measure total pressures of about $1-3 \times 10^{-12}$ mbar. As an alternative, the modified Helmer gauge [13], providing a low pressure limit in the $10^{-14}$ mbar range, might be an interesting additional instrument for future LEIR vacuum applications. In addition, bakeable Pirani and Penning type gauges are used to measure the vacuum during pumpdown and bakeout when the UHV gauges are still switched off. Residual gas analysers are installed for in situ leak detection and to evaluate the gas composition during heavy-ion operation.

The success of the LEIR vacuum system depends highly on the reliability of the bakeout system. A strong emphasis is given to the homogeneity of the bakeout temperature $\left( \pm 10^{\circ} \mathrm{C}\right.$ deviation at $300^{\circ} \mathrm{C}$ ) along the whole machine. Baking at $300^{\circ} \mathrm{C}$ for at least $24 \mathrm{~h}$ is performed to obtain a stainless steel outgassing rate of low $10^{-13}$ to $10^{-14} \mathrm{mbar} \cdot \ell \cdot \mathrm{s}^{-1} \cdot \mathrm{cm}^{-2}$, as required for LEIR. Special shaped vacuum envelopes, tanks, ion pumps, sublimation pumps, and valves are surrounded by heating jackets that were specially manufactured. The bakeout system guarantees a temperature rise of $50^{\circ} \mathrm{C} / \mathrm{h}$, if requested. The vacuum chambers of the four bending magnets and of all quadrupoles are equipped with a second (spare) heating system to be used in case of failure of the original one. As usual, thermocouples are installed around the machine for temperature control and monitoring. 


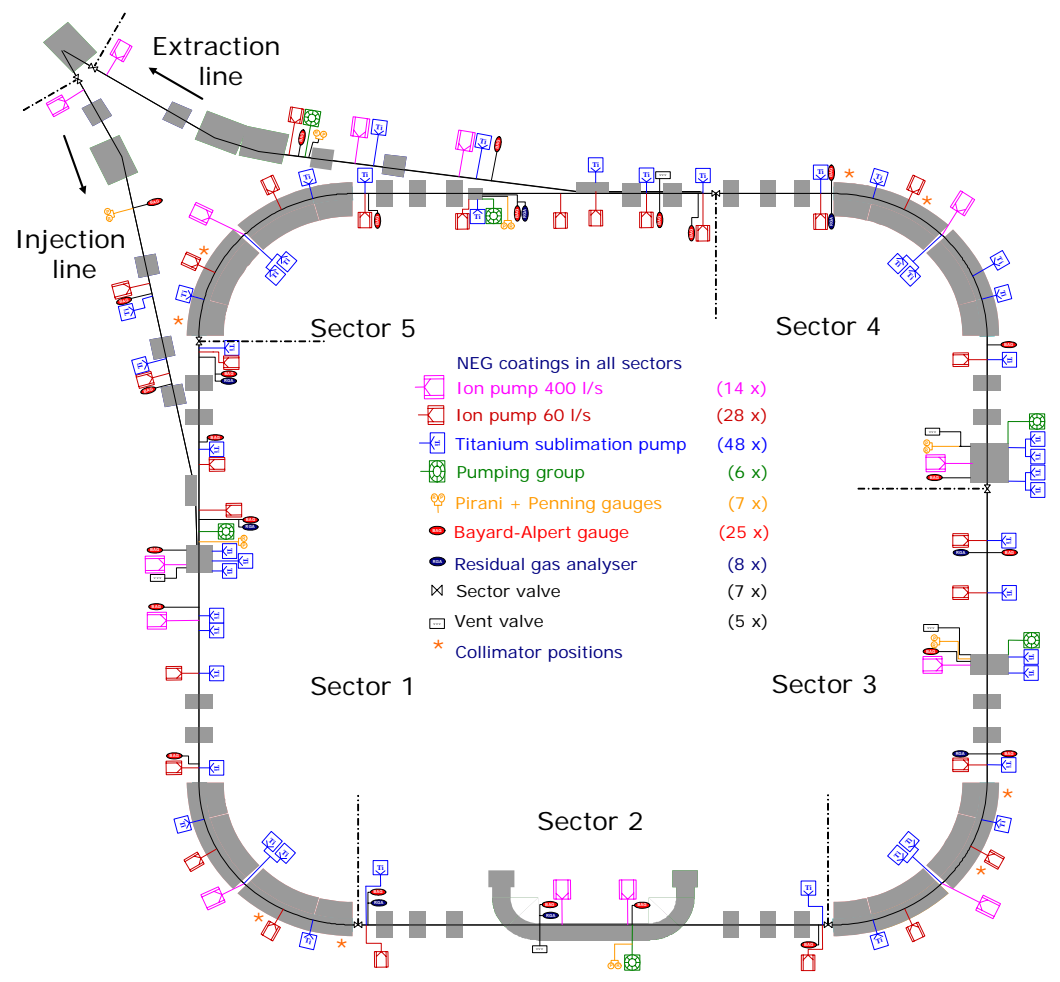

Figure 1: Layout of the LEIR vacuum system showing schematically the injection line, the LEIR main ring, the extraction line and important machine components (in gray), i.e., the four bending magnets, the electron cooler, kicker, septa, and quadrupole magnets. The vacuum system sectorisation, the distribution of pumps, instrumentation, and collimators is displayed (in color).

\section{Challenges for heavy-ion operation and their cures for LEIR}

Heavy-ion induced molecular desorption, currently observed in several particle accelerators world-wide, will also be a critical aspect for the operation of the LEIR vacuum system in the $10^{-12}$ mbar range. Due to electron-capture with the residual gas, $\mathrm{Pb}^{54+}$ ions will be lost as $\mathrm{Pb}^{53+}$ ions during injection (4.2 MeV/u), cooling, accumulation, acceleration (up to $72 \mathrm{MeV} / \mathrm{u}$ ), and extraction. These lost $\mathrm{Pb}^{53+}$ ions will impact under grazing angle onto vacuum chambers and desorb molecules. To quantify and characterize this effect, an intensive experimental program was performed at CERN [2,7,8]. Large desorption yields of $\sim 10^{4}$ molecules/ $\mathrm{Pb}^{53+}$ ion were measured for bare 316LN stainless steel vacuum chambers, which had been vacuum fired at $950^{\circ} \mathrm{C}$ and in situ baked for $24 \mathrm{~h}$ at $300^{\circ} \mathrm{C}$. Some methods to reduce the large pressure rises were found at LINAC 3 and implemented in the LEIR vacuum system design. They are briefly summarized below.

First, coating accelerator-type 316LN stainless steel vacuum chambers with non-evaporable getter (TiZrV) or noble metals (Au, Ag, Pd) reduced the desorption yields by at least one to two orders of magnitude [14]. To obtain very clean surfaces after in situ bakeout and low dynamic gas loads under heavy ion bombardment, the LEIR vacuum system is therefore NEG coated wherever possible.

Second, in each vacuum chamber of the four bending magnets one movable and one fixed collimator (see Figure 2) are placed at locations where impact of the lost $\mathrm{Pb}^{53+}$ ions is expected. 


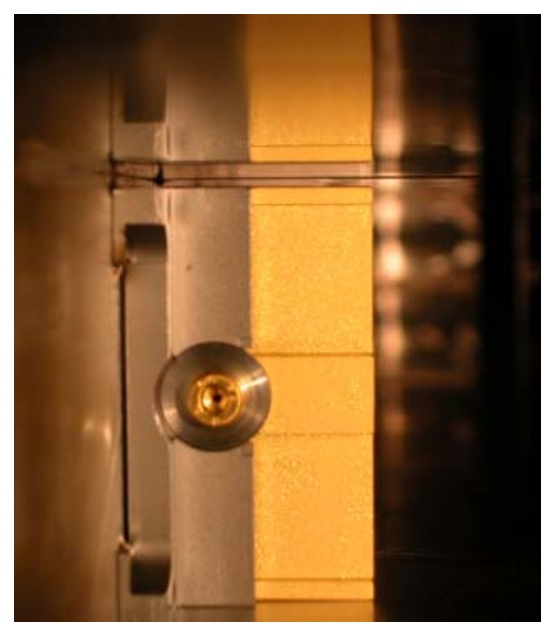

Figure 2: Photograph of a $30 \mu \mathrm{m}$ gold-coated 316LN stainless steel collimator to collect lost $4.2 \mathrm{MeV} / \mathrm{u}$ $\mathrm{Pb}^{53+}$ ions. The collimator is screwed on the back face onto a stainless steel support which is spot-welded at the end of each LEIR bending magnet vacuum chamber.

These collimators are gold-coated stainless steel plates. During LEIR operation, lost ions will hit them under perpendicular impact, which reduces the molecular desorption yield by a factor of $\sim 2$ in comparison to grazing angle bombardment [8]. If necessary, the collimators and their coatings can be exchanged.

Third, the possibility of reducing the dynamic pressure by continuous impact of lost ions (beam scrubbing), demonstrated first at LINAC 3 [2,8,14], may reduce potential pressure rises during LEIR commissioning and operation.

\section{Conclusion}

The dynamic vacuum requirements for LEIR were evaluated and a design was chosen that will cope with the challenge of large dynamic pressure rise due to lost lead ions. The new LEIR ultrahigh vacuum system features a $300^{\circ} \mathrm{C}$ bakeout system, NEG-coated stainless-steel vacuum chambers wherever possible, and low-outgassing collimators to control ion losses. The effect of ion-beam scrubbing during LEIR operation will help to obtain the required gas density for the requested $30 \mathrm{~s}$ beam lifetime. The installation of the vacuum system is ongoing and beam commissioning of LEIR is foreseen to start in August 2005.

\section{Acknowledgements}

The author wants to thank many colleagues working in the AB, AT, and TS departments at CERN, in particular C. Benvenuti, S. Blanchard, P. Chiggiato, J. Hansen, J.-M. Laurent, E. Page, K. Schindl, and P. Strubin for their support, fruitful discussions, and help during the design phase of the LEIR vacuum system. Communications with O. Boine-Frankenheim and T. Stöhlker from GSI (Germany) on cross section data and calculations is also gratefully acknowledged. 


\section{References}

[1] J. Bosser et al., Part. Accel. 63, 171 (1999).

[2] J. Hansen, J.-M. Laurent, N. Madsen, E. Mahner, CERN Report No. LHC/VAC 2001-007 (2001).

[3] A. Krämer, O. Boine-Frankenheim, E. Mustafin, H. Reich-Sprenger, P. Spiller, in Proceedings of European Particle Accelerator Conference, Paris, 2002, p. 2547.

[4] E. Mustafin, O. Boine-Frankenheim, I. Hofmann, H. Reich-Sprenger, P. Spiller, Nucl. Instr. and Methods, A510, No.3 (2003), p.199.

[5] S.Y. Zhang, L.A. Ahrens, in Proceedings of Particle Accelerator Conference, New York, 1999, p. 3294.

[6] S.Y. Zhang et al., in Proceedings of Particle Accelerator Conference, Portland, 2003, p. 54.

[7] E. Mahner, J. Hansen, D. Küchler, M. Malabaila, M. Taborelli, in Proceedings of European Particle Accelerator Conference, Paris, 2002, p. 2568.

[8] E. Mahner, J. Hansen, J.-M. Laurent, N. Madsen, Phys. Rev. ST-AB 6, 013201 (2003).

[9] B. Franzke, IEEE Trans. Nucl. Sci. NS-28, 2116 (1981).

[10] E. Mahner, CERN Report No. LHC/VAC-TN-2002-04 (2002).

[11] L. Ferreira, CERN Reports: Procédure Qualité 867.11, 867.15, 876.16 (2003).

[12] C. Benvenuti, M. Hauer, Nucl. Instr. Methods 140, 453 (1977).

[13] C. Benvenuti, M. Hauer, Le Vide, les Couches Minces 201, 199 (1981).

[14] E. Mahner, J. Hansen, D. Küchler, M. Malabaila, M. Taborelli, Phys. Rev. ST-AB 8, 053201 (2005). 